\title{
Robust Control of the Boost Converter
}

\author{
Domingo Cortés, Joaquín Alvarez, Jaime Alvarez
}

\begin{abstract}
Using the underlying idea of the current-mode control, a new dynamical, sliding-mode control for the boost dc-dc converter is proposed. Such controller is easy to design, robust under load and input voltage variations, exhibits fast response, does not depend on the load (although a knowledge of the load range is necessary to tune the controller), requires only voltage measurements, and can be easily implemented using standard electronic components.
\end{abstract}

\section{INTRODUCTION}

Control of dc-dc converters is a twofold interesting problem. On the one hand, the efficiency, reduced size, low cost and reliability of these devices make them suitable for many applications. They are also circuits which a large number of complex applications are based on. On the other hand, $\mathrm{dc}$-dc converters are discontinuous, nonlinear, nonminimum phase systems, with a highly variable parameter (the load). Motivated by these facts, there has been a continuous effort to design control strategies to improve the performance of power converters. As a result, a considerable number of control schemes based on diverse tools has been proposed. Methods that have been employed ranges from heuristic approaches (see, for example [1], [2], [3]) to well mathematically founded techniques recently proposed (see [4], [5], [6], [7], [8], [9], [10]).

So far, proposed controllers based on nonlinear control theory offer a good performance (see [5] and the references therein for a detailed study and a comparison of some nonlinear control strategies). However, most of them are complex, assume that the load is known, or require nonstandard electronic circuitry to be implemented [5]. This lack of simplicity in implementation makes difficult and expensive to incorporate such control schemes in real applications. As a result, linear techniques and current-mode control, are still the most common tools for control design of dc-dc converters [4]. However, performance of linear techniques is limited. On the other hand, current-mode control has much better performance but its design procedure is not as easy to follow [2], [11].

This paper provides a new dynamical sliding-mode control for the boost dc-dc converter that solves some of the problems above mentioned. The controller is robust under load This
44969

This work was partially supported by CONACYT Mexico under grant

Domingo Cortés and Jaime Alvarez are with Centro de Investigación y Estudios Avanzados del IPN, Departamento de Ingeniería Eléctrica. Av. IPN, 2508, México, D.F. Email: \{dcortes, jalvarez\}@ cinvestav.mx

Joaquín Alvarez is with Centro de Investigación Científica y de Educación Superior de Ensenada, Departamento de Electrónica y Telecomunicaciones. Carretera Tijuana Ensenada km 107, Ensenada B. C. México. Email: jqalvar@cicese.mx and input voltage variations, has a fast response, requires only voltage measurements, and can be easily implemented using standard electronics components. Stability analysis is made considering the nonlinear model of the converter.

The paper is organized as follows. In Section II discontinuous models for the boost converter are presented. These models are used in the following sections. In this section the dc-dc conversion problem is also described. In Section III, the controller is presented. Closed-loop stability is analyzed in Section IV, where some useful expressions for design are obtained. Implementation issues are discussed in Section V. The comments presented in this section provide an easy to follow design procedure. In Section VI simulations results are presented. Conclusions are given in the last section.

\section{THE DC-DC CONVERSION PROBLEM}

\section{A. The switched model}

Figure 1 shows a simplified diagram of the boost converter. The circuit objective is to keep a desired constant output voltage regardless of the input voltage and load variations. From the diagram, a linear model can be obtained for each switch position. Combining both models into a single one yields the following system,

$$
\begin{aligned}
L \frac{d}{d t} z_{1} & =v_{i n}-u z_{2} \\
C \frac{d}{d t} z_{2} & =-\frac{z_{2}}{R}+u z_{1}
\end{aligned}
$$

where $z_{1}$ and $z_{2}$ are the inductor current and the capacitor (output) voltage, respectively. $u$ is a binary variable, $u \in$ $\{0,1\}$, which defines the switch position and plays the role of the control input. The inductor $L$, the capacitor $C$, and the source voltage $v_{\text {in }}$ are suppose to be known constants. The resistance $R$ is unknown but is considered to be constant for analysis purposes.

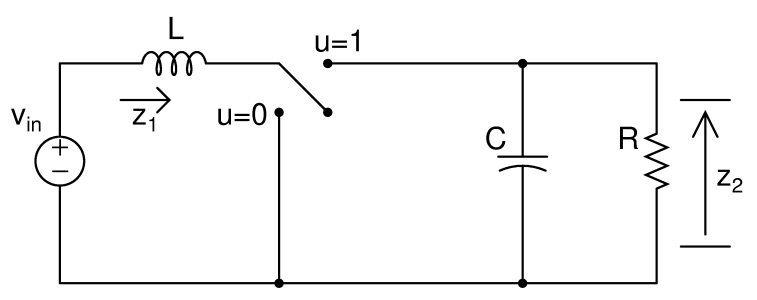

Fig. 1. Schematic diagram of the boost converter. 


\section{B. Normalized model}

Let us consider the coordinate change and time scaling,

$$
x_{1}(\tau)=\frac{1}{v_{\text {in }} \sqrt{C / L}} z_{1}(t), \quad x_{2}(\tau)=\frac{1}{v_{\text {in }}} z_{2}(t), \quad t=\sqrt{L C} \tau
$$

then model (1), is transformed to

$$
\begin{aligned}
& \dot{x}_{1}=1-u x_{2} \\
& \dot{x}_{2}=-\frac{x_{2}}{R_{n}}+u x_{1}
\end{aligned}
$$

where $R_{n}=R \sqrt{C / L} ; R_{n}$ can be seen as a normalized load; it is also known as the circuit quality factor. The dot operator in equations (3) denotes the derivative with respect to the new time $\tau$.

\section{Problem statement}

Because control $u$ (the switch position) is a binary variable changing at a high frequency some variables of the boost converter have discontinuous derivative. Therefore, it is convenient to establish the control objective and some other relationships in terms of average variables. From now on given a function $f(t)$ we regard its average as $\tilde{f}(t)=\frac{1}{T} \int_{t-T}^{t} f(s) \mathrm{d} s$, where $T$ is the switching period.

Before introducing the control goal it is convenient to review some relevant facts about the boost converter. System (1) is said to be in open loop if $u$ is a pulse train independent of system state, with period $T$ and $\tilde{u}=\bar{u}$, where $\bar{u}$ is constant. It is possible to show that in this case the system has an (average) asymptotically stable equilibrium point $\left(\bar{z}_{1}, \bar{z}_{2}\right)=\left(v_{\text {in }} /\left(R \bar{u}^{2}\right), v_{\text {in }} / \bar{u}\right)$. Hence, to obtain $\tilde{z}_{2}=v_{\text {ref }}$ in stationary case, it is just necessary to set $\bar{u}=v_{\text {in }} / v_{\text {ref }}$ in open loop. Note that, since $\bar{u} \in[0,1]$, then $v_{r e f} \geq v_{\text {in }}$. This confirms the well known fact that the boost converter can only amplify its input voltage.

Although the boost converter is open-loop stable its convergence to an equilibrium point may be too slow for most practical applications. Hence, the control objectives are to increase speed of convergence and to make the system robust under load and input voltage variations. Such objectives are not easy to achieve because the circuit is a nonlinear, nonminimum phase system with a highly-varying unknown parameter. (see, for example, [5], [9] for a detailed analysis of dynamical properties of the boost converter). Furthermore, implementation of the controller must be simple enough to keep overall circuit reliability.

The control objective can be established as follows: to design a commutation policy for the switch $u$ such that,

$$
\tilde{z}_{2} \rightarrow v_{\text {ref }}, v_{\text {ref }}>v_{\text {in }}
$$

with a suitable convergence speed even under the presence of load and input voltage variations.

To simplify the analysis, model (3) will be used in the next sections. The control objective for this model is similar to the previous one: to design a commutation policy for $u$ such that,

$$
\tilde{x}_{2} \rightarrow x_{2}^{*}
$$

where $x_{2}^{*}=v_{\text {ref }} / v_{\text {in }}$. Note that $x_{2}^{*}>1$.

\section{THE PROPOSED CONTROLLER}

Given the system (3), we propose the dynamical slidingmode control:

$$
\begin{gathered}
u(\tau)= \begin{cases}0 & \text { if } \sigma(x, \tau)<0 \\
1 & \text { if } \sigma(x, \tau)>0\end{cases} \\
\sigma(x, \tau)=\int_{0}^{\tau}\left(1-u(s) x_{2}(s)\right) \mathrm{d} s \\
+k_{p}\left(x_{2}(\tau)-x_{2}^{*}\right)+k_{i} \int_{0}^{\tau}\left(x_{2}(s)-x_{2}^{*}\right) \mathrm{d} s
\end{gathered}
$$

The underlying idea of such control law is the same that the one behind current-mode control [2]. To see this, note that the first integral term of the sliding surface (7) is an estimated value for the inductor current (see eq. (3a)). Indeed this term and $x_{1}$ only differ by the initial condition of $x_{1}$. In fact what the controller actually does is to make the inductor current equal to the output of a linear function of the voltage error. This is the main idea of current mode control, but expressed as a sliding mode control. Having a well defined mathematical expression for the controller will allow us to formally prove the closed-loop stability of the system.

The controller (6-7) can also be seen as a variation of a controller proposed in [12] using the technique called integral state reconstruction, where the surface

$$
\sigma=\int_{0}^{t}\left(1-u(s) x_{2}(s)\right) \mathrm{d} s-\frac{x_{2}^{* 2}}{R_{n}}+k \int_{0}^{t}\left(x_{2}(s)-x_{2}^{*}(s)\right) \mathrm{d} s
$$

is proposed. Note the similarity between (8) and (7). A significant difference between both surfaces is that (7) does not depend on the load.

The control law (6-7) can also be related to the first sliding-mode control proposed for the boost converter. In [9] it is proposed the sliding surface

$$
\sigma=x_{1}-x_{1}^{*} ; \quad x_{1}^{*}=\frac{x_{2}^{* 2}}{R_{n}}
$$

If in (9) $x_{1}$ and $x_{1}^{*}$ are replaced by $\int_{0}^{t}\left(1-u(s) x_{2}(s)\right) \mathrm{d} s$ and $-k_{p}\left(x_{2}(\tau)-x_{2}^{*}\right)-k_{i} \int_{0}^{\tau}\left(x_{2}(s)-x_{2}^{*}\right) \mathrm{d} s$ respectively, then the controller (6-7) is obtained. That is, surface (7) can be seen as (9) with a different way of assembling $x_{1}$ and $x_{1}^{*}$.

\section{STABILITY}

In this section, stability of the controlled system is proved. During the process useful relationships will be obtained. First let us establish that the system eventually evolves in the sliding surface independently of its initial condition.

Lemma 1: Let us consider system (3) with the control (67). If

$$
0<k_{i}<\frac{1}{x_{2}^{*}}, 0<k_{i}-\frac{k_{p}}{R_{n}}<1
$$

Then any trajectory of the system goes into a sliding movement on $\sigma(x, \tau)=0$. 
Proof: Consider the extended space $X_{e}=\left(x_{1}, x_{2}, \sigma\right)$. According to (7), the third state equation of the extended system is

$$
\dot{\sigma}=1-u x_{2}+k_{p}\left(u x_{1}-\frac{x_{2}}{R_{n}}\right)+k_{i}\left(x_{2}-x_{2}^{*}\right)
$$

The proof is divided in two parts. First it will be shown that there is a subset $\mathscr{S}$ of $X_{e}$ where $\sigma \dot{\sigma}<0$ and $0<u_{e q}<1$ accomplish, that is a sliding mode can exist. Then, it will be shown that any trajectory hits the surface $\sigma=0$ within this set.

Consider the two cases:

Case 1: $\sigma<0$. In this case, $u=0$ and $\dot{\sigma}$ becomes

$$
\dot{\sigma}=\left(k_{i}-\frac{k_{p}}{R_{n}}\right) x_{2}+\left(1-k_{i} x_{2}^{*}\right)
$$

Hence, if $\sigma<0$ then $\dot{\sigma}>0$ in the set

$$
\left\{x \in \mathfrak{R}^{2} \mid\left(k_{i}-\frac{k_{p}}{R_{n}}\right) x_{2}+\left(1-k_{i} x_{2}^{*}\right)>0\right\}
$$

Case 2: $\sigma>0$. In such a case, $u=1$ and $\dot{\sigma}$ becomes

$$
\dot{\sigma}=\left(k_{p} x_{1}-x_{2}\right)+\left(k_{i}-\frac{k_{p}}{R_{n}}\right) x_{2}+\left(1-k_{i} x_{2}^{*}\right)
$$

Therefore, if $\sigma>0$ then $\dot{\sigma}<0$ in the set

$$
\left\{x \in \mathfrak{R}^{2} \mid x_{2}-k_{p} x_{1}>\left(k_{i}-\frac{k_{p}}{R_{n}}\right) x_{2}+\left(1-k_{i} x_{2}^{*}\right)\right\}
$$

Combining both cases, $\sigma \dot{\sigma}<0$ in the intersection of sets (13) and (15), that is, in the set

$$
\mathscr{S}=\left\{x \in \mathfrak{R}^{2} \mid 0<\left(k_{i}-\frac{k_{p}}{R_{n}}\right) x_{2}+\left(1-k_{i} x_{2}^{*}\right)<x_{2}-k_{p} x_{1}\right\}
$$

From (11) the equivalent control, (see [13]) results in

$$
u_{e q}=\frac{\left(k_{i}-\frac{k_{p}}{R_{n}}\right) x_{2}+\left(1-k_{i} x_{2}^{*}\right)}{x_{2}-k_{p} x_{1}}
$$

Note that, $0<u_{e q}<1$ in the set $\mathscr{S}$.

Since $\sigma \dot{\sigma}<0$ and $0<u_{e q}<1$ in the set $\mathscr{S}$, a sliding movement takes place when the system trajectory hits the surface $\sigma=0$ within this set.

It will be shown now that any system trajectory hits the surface $\sigma=0$ within the set $\mathscr{S}$. Let us suppose that, at a certain time, $\sigma(x)<0$. In this case $u=0$ and the system equations are given by,

$$
\begin{aligned}
& \dot{x}_{1}=1 \\
& \dot{x}_{2}=-\frac{x_{2}}{R_{n}}
\end{aligned}
$$

then, $x_{2} \rightarrow 0$, and from (11)

$$
\dot{\sigma} \rightarrow 1-k_{i} x_{2}^{*}
$$

Then, by conditions (10), $\sigma(x)$ eventually increases, and goes through zero hitting the surface. On the contrary, if $\sigma(x)>0$ then $u=1$ and the system equations are given by,

$$
\begin{aligned}
& \dot{x}_{1}=1-x_{2} \\
& \dot{x}_{2}=-\frac{x_{2}}{R_{n}}+x_{1}
\end{aligned}
$$

therefore $x \rightarrow\left(1 / R_{n}, 1\right)$, and,

$$
\dot{\sigma} \rightarrow k_{i}\left(1-x_{2}^{*}\right)
$$

Since $x_{2}^{*}>1$ then $\sigma(x)$ eventually decreases and goes through zero. That is, the trajectory also hits the surface in this case. Hence, we can conclude that the trajectory reaches the surface (7) from any point in $\left(x_{1}, x_{2}\right) \in \mathfrak{R}^{2}$. Besides, the system trajectory moves towards the set $\mathscr{S}$, independently of the sign of $\sigma$. Therefore, any trajectory of the system hits the surface $\sigma(x)=0$ within the set $\mathscr{S}$ entering in an sliding regime.

The set $\mathscr{S}$ defines the region on the plane $\left(x_{1}, x_{2}\right)$ where a sliding mode can exist. It can be rewritten as

$$
\mathscr{S}=\left\{x \in \mathfrak{R}^{2} \mid x_{2}>-\frac{1-k_{i} x_{2}^{*}}{k_{i}-\frac{k_{p}}{R_{n}}}, x_{2}>\frac{k_{p} x_{1}+1-k_{i} x_{2}^{*}}{1-\left(k_{i}-\frac{k_{p}}{R_{n}}\right)}\right\}
$$

and it is the shaded area depicted in Figure 2.

According to equivalent control method [13], a model to describe the system trajectory when it evolves on the sliding surface can be found by substituting $u$ by $u_{e q}$ in (3). Hence, on the sliding surface the trajectory is governed by

$$
\begin{aligned}
& \dot{x}_{1}=\frac{-k_{p}\left(x_{1}-\frac{x_{2}^{2}}{R_{n}}\right)-k_{i} x_{2}\left(x_{2}-x_{2}^{*}\right)}{x_{2}-k_{p} x_{1}} \\
& \dot{x}_{2}=\frac{\left(x_{1}-\frac{x_{2}^{2}}{R_{n}}\right)+k_{i} x_{1}\left(x_{2}-x_{2}^{*}\right)}{x_{2}-k_{p} x_{1}}
\end{aligned}
$$

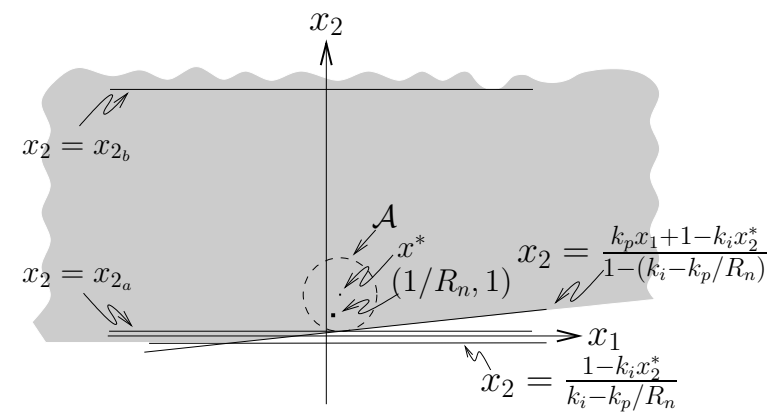

Fig. 2. Zone where the sliding movement can exist.

Remark 1: Lemma 1 does not assert that if the system trajectory goes into the sliding mode on the surface $\sigma=0$ then it remains there. The trajectory could leave the sliding mode, but if this happens, it eventually returns to the surface. Furthermore, the trajectory can only leave the sliding surface towards the $\sigma>0(u=1)$ side. This can be shown by making $u_{e q} \rightarrow 0$, that is, $x_{2} \rightarrow-\left(1-k_{i} x_{2}^{*}\right) /\left(k_{i}-k_{p} / R_{n}\right)$ in (23) which results in,

$$
\dot{x}_{1} \rightarrow 1, \dot{x}_{2} \rightarrow \frac{1-k_{i} x_{2}^{*}}{R_{n}\left(k_{i}-\frac{k_{p}}{R_{n}}\right)}
$$

Hence, when $u_{e q} \rightarrow 0$, the trajectory moves towards the set $\mathscr{S}$ and the system cannot leave the sliding mode towards the $\sigma<0(u=0)$ side. With reference to Figure 2, the previous argument means that if the system is on a sliding 
regime, a trajectory cannot leave the sliding mode crossing the horizontal line $x_{2} \rightarrow-\left(1-k_{i} x_{2}^{*}\right) /\left(k_{i}-k_{p} / R_{n}\right)$.

Theorem 1: If condition (10) holds then the point $x_{d}=\left(x_{2}^{* 2} / R_{n}, x_{2}^{*}\right)$ is an asymptotically stable equilibrium point of the system (3) controlled by (6-7).

Proof: When the trajectory is on the sliding regime, the system is governed by (23). Let the function,

$$
V(x)=\frac{1}{2}\left(e_{1}^{2}+e_{2}^{2}\right)
$$

where

$$
e_{1}=x_{1}-\frac{x_{2}^{*^{2}}}{R_{n}}, e_{2}=x_{2}-x_{2}^{*}
$$

be a Lyapunov function candidate. The derivative of $V(x)$ along the trajectory of the system when it evolves on the sliding surface can be written as,

$$
\dot{V}(x)=-\frac{\left(a e_{1}^{2}+b e_{1} e_{2}+c e_{2}^{2}\right)}{x_{2}-k_{p} x_{1}}
$$

with

$a=k_{p}, b=-\frac{k_{p}}{R_{n}}\left(x_{2}+x_{2}^{*}\right)-\left(1-k_{i} x_{2}^{*}\right), c=\frac{x_{2}+x_{2}^{*}-k_{i} x_{2}^{*^{2}}}{R_{n}}$

From (16), $x_{2}-k_{p} x_{1}>0$ in the set $\mathscr{S}$, hence, for $\dot{V}(x)$ to be negative in the set $\mathscr{S},\left(a e_{1}^{2}+b e_{1} e_{2}+c e_{2}^{2}\right)$ must be positive, which is assured in the region where $4 a c-b^{2}>0$ holds. That is, in the region,

$$
\begin{aligned}
& \frac{k_{p}^{2}}{R_{n}^{2}} x_{2}^{2}-\frac{2 k_{p}}{R_{n}}\left(1+k_{i} x_{2}^{*}-\frac{k_{p} x_{2}^{*}}{R_{n}}\right) x_{2}+ \\
& \quad\left(1-k_{i} x_{2}^{*}\right)^{2}+\frac{k_{p} x_{2}^{*}}{R_{n}}\left(\frac{k_{p} x_{2}^{*}}{R_{n}}-2\left(1-k_{i} x_{2}^{*}\right)\right)<0
\end{aligned}
$$

Inequality (29) is accomplished in the set,

$$
\mathscr{U}=\left\{x: x_{2_{a}}<x_{2}<x_{2_{b}}\right\}
$$

where $x_{2_{a}}$ and $x_{2_{b}}$ are given by

$$
\begin{aligned}
& x_{2_{a}}=\frac{R_{n}}{k_{p}}\left(1-x_{2}^{*}\left(k_{i}-\frac{k_{p}}{R_{n}}\right)\right)-2 R_{n} \sqrt{k_{i} x_{2}^{*}\left(1-\frac{k_{p}}{R_{n}} x_{2}^{*}\right)} \\
& x_{2_{b}}=\frac{R_{n}}{k_{p}}\left(1-x_{2}^{*}\left(k_{i}-\frac{k_{p}}{R_{n}}\right)\right)+2 R_{n} \sqrt{k_{i} x_{2}^{*}\left(1-\frac{k_{p}}{R_{n}} x_{2}^{*}\right)}
\end{aligned}
$$

Note that condition (10) implies $x_{2_{a}}$ and $x_{2_{b}}$ are real.

Since $\dot{V}(x)$ is negative in the set

$$
\mathscr{N}=\left\{x \in \mathfrak{R}^{2} \mid x \in \mathscr{U} \cap \mathscr{S}\right\}
$$

which contains $x^{*}$, then $x^{*}$ is an asymptotically stable equilibrium point.

Furthermore, let us define the following set,

$$
\mathscr{A}=V(x)<r
$$

where $r$ is the largest number such that $\mathscr{A} \subset \mathscr{N}$. If the system trajectory hits the sliding surface within the set $\mathscr{A}$ then the trajectory remains on the sliding surface thereafter and tends asymptotically to the point $x^{*}[14]$.
Remark 2: If the trajectory hits the sliding surface on a point not belonging to $\mathscr{A}$ but in $\mathscr{N}$, the trajectory still converges to the point $x_{d}$ but could leave the sliding mode before reaching the set $\mathscr{A}$. If the trajectory leaves the sliding surface, it will return (see Remark 1). Furthermore, when the trajectory leaves the sliding surface, $u=1$, then the next time it hits the surface it will be closer to the point $\left(1 / R_{n}, 1\right)$. Hence, if $\left(1 / R_{n}, 1\right) \in \mathscr{A}$ then the trajectory eventually hits the sliding surface within the set $\mathscr{A}$. Summing up, the set $\mathscr{N}$ is a region of attraction of $x_{d}$ provided that $\left(1 / R_{n}, 1\right) \in \mathscr{A}$.

The sets introduced in the stability proof are depicted in Figure 2. As it will be shown in Section VI, the set $\mathscr{N}$ covers a significant part of the first quadrant of the $\left(x_{1}, x_{2}\right)$ plane for practical examples.

\section{IMPLEMENTATION ISSUES}

Due to its reduced number of parameters, the normalized model can also be useful to select control parameters. Hence, to tune control parameters for a given boost converter it is convenient to obtain its normalized model. Since the worst case in terms of stability happens when $R_{n}$ is small, the normalized model should be obtained using the minimum expected load.

Having the normalized model, control parameters can be set as follows. First, set $k_{p}=0$ and then adjust $k_{i}$ to get an adequate overshoot. In view of conditions (10), $k_{i}=$ $(1 / 3)\left(1 / x_{2}^{*}\right)$ is a good starting value. Then increase $k_{p}$ until a desirable performance is obtained. In this step readjust of $k_{i}$ might be necessary. During this procedure keep on mind that conditions (10) must hold to guarantee closed-loop stability.

Once normalized control parameters are selected, the nonnormalized expression for the controller can be obtained by reversing transformation (2). This results in the following control law for system (1)

$$
\begin{gathered}
u(t)= \begin{cases}0 & \text { if } \sigma(x, t)<0 \\
1 & \text { if } \sigma(x, t)>0\end{cases} \\
\sigma(z, t)=\left(\frac{1}{v_{i n} \sqrt{L C}}\right)\left[\int_{0}^{t}\left(v_{i n}-u(s) z_{2}(s)\right) d s\right. \\
\left.+\sqrt{L C} k_{p}\left(z_{2}(s)-v_{r e f}\right)+k_{i} \int_{0}^{t}\left(z_{2}(s)-v_{r e f}\right) d s\right]
\end{gathered}
$$

The right-hand side (rhs) of (36) can be scaled without modifying the sliding surface and consequently, without modifying overall closed-loop system performance. Multiplying (rhs) of (36) times $G v_{\text {in }} \sqrt{L C}$, results in

$$
\begin{aligned}
& \sigma(z, t)=G \int_{0}^{t}\left(v_{\text {in }}-u(s) z_{2}(s)\right) d s \\
& +G \sqrt{L C} k_{p}\left(z_{2}(t)-v_{r e f}\right)+G k_{i} \int_{0}^{t}\left(z_{2}(s)-v_{r e f}\right) d s
\end{aligned}
$$

where $G$ is an arbitrary constant. Introduction of $G$ allows us to scale measures to keep control signals manageable.

A constant switching frequency is achieved for the controller (35),(37) if a hysteresis loop is introduced in (35) (see Figure 3). Such hysteresis loop creates a boundary layer 
preventing the switch changes of position while $|\sigma|<h / 2$ [13]. That is $h=\Delta \sigma$, where $\Delta \sigma$ is the width of the boundary layer.

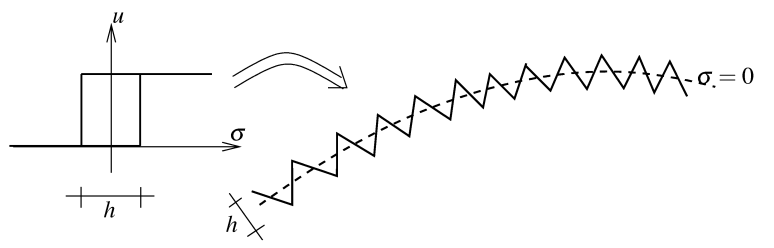

Fig. 3. A hysteresis loop introduces a boundary layer limiting the switching frequency.

A relationship between $h$ and the switching period can be obtained. To this end, note that when $\tilde{z}_{2}=v_{\text {ref }}$ (stationary case) then from (37), $\dot{\sigma}=G\left(v_{i n}-u z_{2}\right)=G L \dot{z}_{1}$. Hence $h=$ $G L \Delta z_{1}$, where $\Delta z_{1}$ is the current ripple. Furthermore, from (1a)

$$
\dot{z}_{1} \approx \frac{v_{i n}}{L}-u v_{r e f}
$$

From (38) and with reference to Figure 4 when $u=0$ (that is when $\left.t \in\left(k T, K T+t_{1}\right]\right)$ then $z_{1} \approx \frac{v_{\text {in }}}{L}(t-k T)+z_{1 l}$ thus

$$
\left.\Delta z_{1}=\left|z\left(k T+t_{1}\right)-z(k T)\right| \approx \frac{v_{i n}}{L} t_{1}\right)
$$

On the other hand when $u=1$ (that is when $t \in\left(k T+t_{1},(k+\right.$ 1)T]) then $z_{1} \approx \frac{v_{\text {in }}-v_{\text {ref }}}{L}\left(t-\left(k T+t_{1}\right)\right)+z_{1 u}$, hence

$$
\Delta z_{1}=\left|z(k T+T)-z\left(k T+t_{1}\right)\right| \approx \frac{v_{r e f}-v_{i n}}{L}\left(T-t_{1}\right)
$$

From (39) and (40) results,

$$
T \approx \frac{v_{r e f}}{v_{\text {in }}\left(v_{\text {ref }}-v_{\text {in }}\right)} L \Delta z_{1} \approx \frac{v_{\text {ref }}}{v_{\text {in }}\left(v_{\text {ref }}-v_{\text {in }}\right)} \frac{h}{G}
$$

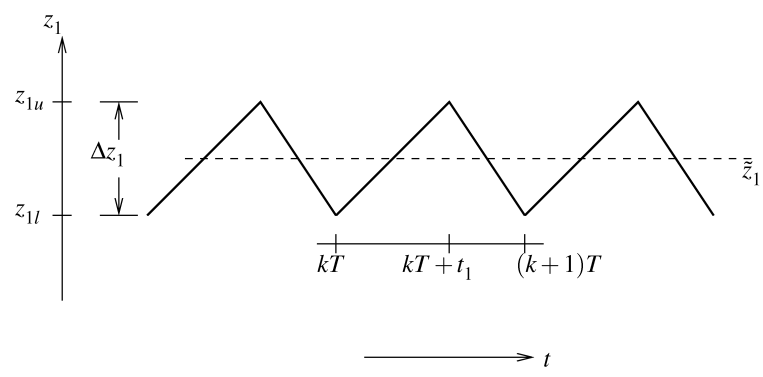

Fig. 4. Close view of the current ripple.

Figure 5 shows a block diagram of the controlled system.

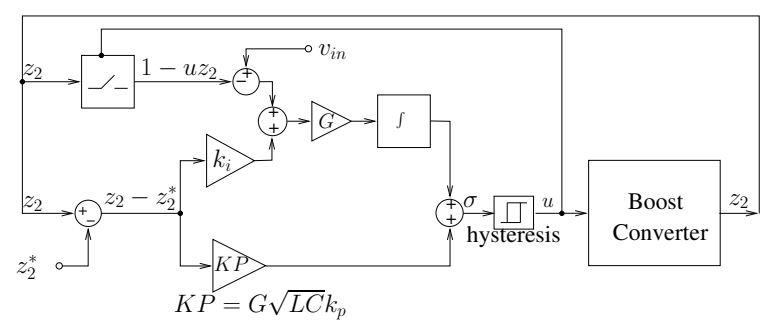

Fig. 5. Block diagram of the controlled converter.

\section{Simulation RESUlts}

To illustrate the performance of the proposed controller, it will be applied to a boost converter with the following parameters: $L=0.36 \mathrm{mH}, C=28.2 \mu \mathrm{F}, v_{i n}=48 \mathrm{~V}$, and a minimum load of $48 \Omega$. The output voltage to be regulated is $96 \mathrm{~V}$, so the duty cycle of $u$ in stationary state should be $50 \%$.

The normalized load of this converter results in $R_{n}=$ 13.43. Following the procedure described in the previous section, $k_{i}=0.1$ and $k_{p}=0.5$ were found to be appropriate for controlling the normalized model. For simulation purposes we set $G=1$. Relation (41) can be used to calculate $h$ in order to get a switching frequency of $30 \mathrm{KHz}$. This results in $h=0.0016$.

Figure 6 and 7 show the performance of the controller compared against two controllers which are representative of nonlinear controllers proposed recently. In Figure 6 control $(35,37)$ is compared with the average passivity-based control law proposed in [10] given by

$$
\begin{aligned}
\dot{\tilde{u}}= & \frac{\tilde{u}}{C\left(v_{\text {in }}+\left(\tilde{z}_{1}-z_{1 d} R_{1}\right)\right)} \\
& \left(\tilde{u}^{2} z_{1 d}-\frac{v_{i n}+\left(z_{1}-z_{1 d}\right) R_{1}}{R}-\frac{R_{1} C}{L}\left(v_{\text {in }}-\tilde{u} z_{2}\right)\right)
\end{aligned}
$$

where $z_{1 d}=\frac{v_{r e f}^{2}}{R v_{i n}}$ and $R_{1}>0$ is a control design parameter.

In Figure 7 comparison is made against the average control law

$$
\tilde{u}=\frac{1}{v_{\text {ref }}}\left(\frac{\tilde{z}_{2}}{v_{\text {ref }}}\right)^{\alpha} ; \quad 0<\alpha<1
$$

proposed in [8].

To examine the performance of controllers under load variations sudden changes of the load were introduced in simulations. At $t=5 \mathrm{mS}$ the load is changed from $48 \Omega$ to $24 \Omega$. And a $t=10 \mathrm{mS}$ the load is changed again from $24 \Omega$ to $96 \Omega$.

It can be seen from Figure 6 that the settling time of the controller we propose is as fast as the one obtained by (42). However the controller proposed here rapidly restore the output voltage to its desired value whereas the controller (42) can not compensate the load changes.

The nonlinear control law (43) is easy to implement and robust under load variation. However, as Figure 7 shows, its settling time is far more slow than the one proposed here.

We must point out that in simulations of Figures 6 and 7 the controller parameters $k_{p}$ and $k_{i}$ were chosen to keep small the output voltage variation under load changes. However if the maximum current is a concern, more conservative controller parameters may be used.

\section{CONCLUSIONS}

The key idea of current-mode control has been employed to design a new dynamical sliding-mode control. The proposed controller has some properties that made current mode control so popular as fast settling time, robustness under 


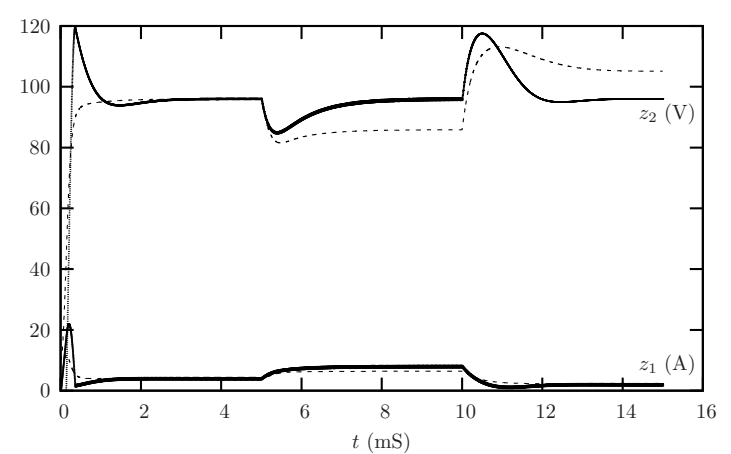

Fig. 6. Performance of the controller proposed.

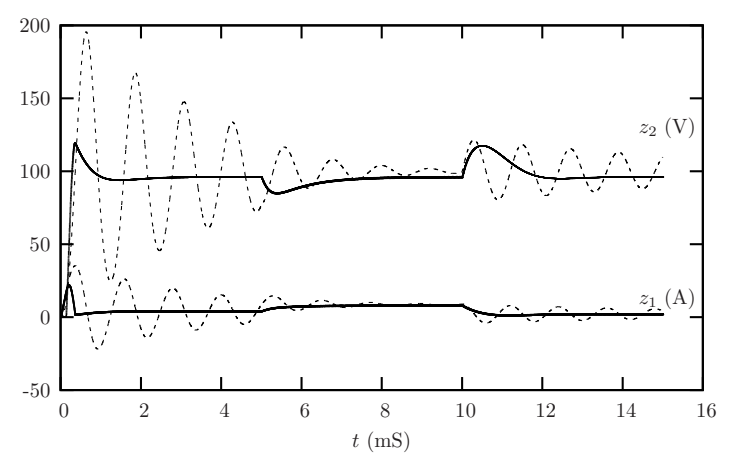

Fig. 7. Comparison with another nonlinear robust controller.

load and input voltage variations and easy implementation. In addition, the controller proposed have a precise mathematical definition which allowed us to carry out a stability analysis. Some expressions found in the stability analysis lead us to set out an easy to follow design procedure.

The controller performance was examined in simulation and comparison with others nonlinear controllers was presented. Simulations showed the excellent robustness of the controller under load and input voltage variations. A practical implementation of the controller is being developed and its results will be reported elsewhere.

A simulation to generate time-variable signals with this controller has been performed obtaining good results. However, a further analysis is necessary to provide a solid theoretical foundation for this application.

\section{REFERENCES}

[1] R. D. Middlebrook, "Modeling current-programmed buck and boost regulators," IEEE Trans. on Power Electronics, vol. 4, no. 1, pp. 3652, January 1989.

[2] R. B. Ridley, "A new continuous-time model fro current-mode control," IEEE Trans. on Power Electronics, vol. 6, no. 2, pp. 271-280, april 1991.

[3] K. Smedley, "One-cycle control of switching converters," IEEE transactions on power electronics, vol. 10, no. 6, pp. 625-633, 1995.

[4] J. Alvarez-Ramirez, G. Espinosa-Pérez, and D. Noriega-Pineda, "Current-mode control of dc-dc power converters a backstepping approach," in Proceedings of the 2001 IEEE International Conference on Control Applications, September 2001, pp. 190-195.

[5] G. Escobar, R. Ortega, H. Sira-Ramírez, J.-P. Vilain, and I. Zein, "An experimental comparison of several nonlinear controllers for power converters," Control System Magazine, no. 2, pp. 68-82, 1999.

[6] A. Kugi and K. Schlacher, "Nonlinear $H_{\infty}$ controller design for dc-todc power converters," IEEE Trans. on Ind. Elect., vol. 7, no. 2, pp. 230-237, 1999.

[7] F. Leung, P. Tam, and C. Li, "An improved LQR-based controller for switching dc-dc converters," IEEE Trans. on Ind. Elect., vol. 40, no. 5, pp. 521-528, 1993.

[8] H. Rodriguez, R. Ortega, G. Escobar, and B. Nikita, "A robustly stable output feedback saturated controller for the boost dc-to-dc converter," System \& Control Letters, vol. 40, 2000.

[9] H. Sira-Ramírez, "Sliding motions in bilinear switched networks," IEEE Trans. on Cir. and Syst., vol. 34, no. 8, pp. 919-933, 1987.

[10] H. Sira-Ramírez, R. Perez-Moreno, R. Ortega, and M. Garcia-Esteban, "Passivity-based controller for stabilization of dc-to-dc power converters," Automatica, vol. 33, pp. 499-513, 1997.

[11] D. Kimhi and S. Ben-Yaakov, "A spice model for current mode pwm converters operating under continuous inductor current conditions," IEEE Trans. on Power Electronics, vol. 6, no. 2, pp. 281-286, Appril 1991.

[12] H. Sira-Ramírez, R. Marquez-Contreras, and M. Flies, "Sliding mode control of dc-to-dc power converters using integral reconstructors," Int. J. Robust Nonlinear Control, vol. 12, pp. 1173-1186, 2002.

[13] V. Utkin, Sliding Modes in Control and Optimization. Springer Verlag, 1992.

[14] J. P. La Salle and S. Lefschetz, "Stability by Lyapunov direct methods with applications," in Mathematics in Science and engeering. Academic Press, 1961, vol. 4. 